VOL. $71(2005) \quad[353-358]$

\title{
A SIMPLE PROOF OF AN EXPANSION OF AN ETA-QUOTIENT AS A LAMBERT SERIES
}

\section{SHAUN COOPER}

We give a simple proof of the identity

$$
\prod_{n=1}^{\infty} \frac{\left(1-q^{3 n}\right)^{10}}{\left(1-q^{n}\right)^{3}\left(1-q^{9 n}\right)^{3}}=1+3 \sum_{\substack{n=1 \\ 9 \gamma_{n}}}^{\infty} \frac{n q^{n}}{1-q^{n}} .
$$

The proof uses only a few well-known properties of the cubic theta functions $a(q)$, $b(q)$ and $c(q)$. We show this identity implies the interesting definite integral

$$
\int_{0}^{e^{-2 \pi / 3}} \prod_{n=1}^{\infty} \frac{\left(1-q^{3 n}\right)^{10}}{\left(1-q^{n}\right)^{6}} d q=\frac{1}{3 \sqrt{3}}
$$

\section{INTRODUCTION}

The purpose of this article is to give a direct proof of the following identity.

THEOREM 1.1. Let $q$ be a complex number satisfying $|q|<1$. Then

$$
\prod_{n=1}^{\infty} \frac{\left(1-q^{3 n}\right)^{10}}{\left(1-q^{n}\right)^{3}\left(1-q^{9 n}\right)^{3}}=1+3 \sum_{\substack{n=1 \\ g / n}}^{\infty} \frac{n q^{n}}{1-q^{n}}
$$

The summation is over all positive integers $n$ excluding multiples of 9 .

This result was discovered using symbolic computation by Borwein and Garvan [4], and it was used to produce a ninth order iteration that converges to $1 / \pi$. The proof of Theorem 1.1 in [4] appeals to two entries in Ramanujan's Notebook [16, Chapter 20 Entry 1(iv) and Chapter 21 Entry 7(i)]. The proofs of these entries in Berndt's excellent book [1] take several pages, and appeal to several earlier results in Ramanujan's Notebook.

Two proofs of Theorem 1.1 were given by Berndt, Chan, Liu and Yesilyurt [3]. The first is essentially the same as the one in [4]. The second proof in [3] uses less sophisticated machinery, but is more than three pages long, and depends on another entry in Ramanujan's Notebook [16, Chapter 20 Entry 1(v)].

Received May 9, 2005

Copyright Clearance Centre, Inc. Serial-fee code: 0004-9727/05 \$A2.00+0.00. 
Another proof of Theorem 1.1 of a completely different nature was obtained by Farkas and Kra [9, p. 307]. Their proof uses meromorphic functions defined on Riemann surfaces.

In view of the importance of Theorem 1.1, it is desirable to have as direct a proof as possible. We give such a proof, which depends only on the well-known properties satisfied by the cubic theta functions $a(q), b(q)$ and $c(q)$ given in Lemma 2.1 below.

We conclude by showing that Theorem 1.1 leads to an evaluation of a definite integral. Three similar integrals were given by Fine [10, pp. 86-91].

\section{PROOF}

The three cubic theta functions are defined by

$$
\begin{aligned}
a(q) & =\sum_{m} \sum_{n} q^{m^{2}+m n+n^{2}}, \\
b(q) & =\sum_{m} \sum_{n} q^{m^{2}+m n+n^{2}} \omega^{m-n}, \\
c(q) & =\sum_{m} \sum_{n}^{n} q^{(m+1 / 3)^{2}+(m+1 / 3)(n+1 / 3)+(n+1 / 3)^{2}},
\end{aligned}
$$

where $\omega=\exp (2 \pi i / 3)$ and $q=e^{-2 \pi t}, \operatorname{Re}(t)>0$. The summation indices $m$ and $n$ range over all integer values. The following are some well known properties of the cubic theta functions.

LEMMA 2.1.

$$
\begin{aligned}
a(q)^{3} & =b(q)^{3}+c(q)^{3}, \\
b(q) & =\prod_{n=1}^{\infty} \frac{\left(1-q^{n}\right)^{3}}{\left(1-q^{3 n}\right)}, \\
c(q) & =3 q^{1 / 3} \prod_{n=1}^{\infty} \frac{\left(1-q^{3 n}\right)^{3}}{\left(1-q^{n}\right)}, \\
a(q) & =a\left(q^{3}\right)+2 c\left(q^{3}\right), \\
b(q) & =a\left(q^{3}\right)-c\left(q^{3}\right), \\
a(q) & =1+6 \sum_{n=1}^{\infty}\left(\frac{q^{3 n-2}}{1-q^{3 n-2}}-\frac{q^{3 n-1}}{1-q^{3 n-1}}\right), \\
a(q)^{2} & =1+12 \sum_{\substack{n=1 \\
3 / n}}^{\infty} \frac{n q^{n}}{1-q^{n}} .
\end{aligned}
$$

Equation (2.2) was discovered and proved by Borwein and Borwein [5]. Additional proofs have since been given by Borwein, Borwein and Garvan [6], Chapman [7], Garvan [11], Hirschhorn, Garvan and Borwein [12], Liu [14] and Solé [18]. Proofs of (2.3)-(2.6) 
can be found in $[6,11,12]$. Equation (2.7) was known to Lorenz and Ramanujan; see [13]. A beautiful and elementary proof of (2.8) using (2.7) was given by Ramanujan [15, equation 19].

Lemma 2.9. Let $x=c(q)^{3} / a(q)^{3}, z=a(q), X=c\left(q^{3}\right)^{3} / a\left(q^{3}\right)^{3}, Z=a\left(q^{3}\right)$. Then

$$
\begin{aligned}
x & =1-\left(\frac{1-X^{1 / 3}}{1+2 X^{1 / 3}}\right)^{3}, \\
z & =Z\left(1+2 X^{1 / 3}\right) \\
X & =\left(\frac{1-(1-x)^{1 / 3}}{1+2(1-x)^{1 / 3}}\right)^{3}, \\
Z & =\frac{z}{3}\left(1+2(1-x)^{1 / 3}\right) .
\end{aligned}
$$

Proof: From Lemma 2.1 we have

$$
\begin{aligned}
1-x & =1-\frac{c(q)^{3}}{a(q)^{3}} \\
& =\frac{b(q)^{3}}{a(q)^{3}} \\
& =\left(\frac{a\left(q^{3}\right)-c\left(q^{3}\right)}{a\left(q^{3}\right)+2 c\left(q^{3}\right)}\right)^{3} \\
& =\left(\frac{1-X^{1 / 3}}{1+2 X^{1 / 3}}\right)^{3}
\end{aligned}
$$

This proves the first part. Similarly,

$$
\begin{aligned}
z & =a(q) \\
& =a\left(q^{3}\right)+2 c\left(q^{3}\right) \\
& =a\left(q^{3}\right)\left(1+2 \frac{c\left(q^{3}\right)}{a\left(q^{3}\right)}\right) \\
& =Z\left(1+2 X^{1 / 3}\right) .
\end{aligned}
$$

This proves the second part. The third and fourth parts are obtained by rearranging the first two parts and solving for $X$ and $Z$.

REMARK 2.10. The first two formulas in Lemma 2.9 are called the trimidiation formulas, and the last two are called the triplication formulas. See [2, pp. 101-102] for another proof and further explanation. 
Proof OF THEOREM 1.1: Using Lemmas 2.1 and 2.9, we have

$$
\begin{aligned}
1+3 \sum_{\substack{n=1 \\
\otimes \psi_{n}}}^{\infty} \frac{n q^{n}}{1-q^{n}} & =\frac{1}{4}\left(a(q)^{2}+3 a\left(q^{3}\right)^{2}\right) \\
& =\frac{1}{4}\left(z^{2}+\frac{z^{2}}{3}\left(1+2(1-x)^{1 / 3}\right)^{2}\right) \\
& =\frac{z^{2}}{3}\left(1+(1-x)^{1 / 3}+(1-x)^{2 / 3}\right) \\
& =\frac{z^{2} x}{3\left(1-(1-x)^{1 / 3}\right)} \\
& =\frac{c(q)^{3}}{3 a(q)(1-b(q) / a(q))} \\
& =\frac{c(q)^{3}}{3(a(q)-b(q))} \\
& =\frac{c(q)^{3}}{9 c\left(q^{3}\right)} \\
& =\prod_{n=1}^{\infty} \frac{\left(1-q^{3 n}\right)^{10}}{\left(1-q^{n}\right)^{3}\left(1-q^{9 n}\right)^{3}} .
\end{aligned}
$$

\section{A DEFinite integral}

In this section we state and prove the value of an interesting definite integral. We use the same method of proof as Fine [10, pp. 86-91], who gave three similar integrals.

THEOREM 3.1 .

$$
\int_{0}^{e^{-2 \pi / 3}} \prod_{n=1}^{\infty} \frac{\left(1-q^{3 n}\right)^{10}}{\left(1-q^{n}\right)^{6}} d q=\frac{1}{3 \sqrt{3}} .
$$

Proof: From Theorem 1.1 we have

$$
\prod_{n=1}^{\infty} \frac{\left(1-q^{3 n}\right)^{10}}{\left(1-q^{n}\right)^{3}\left(1-q^{9 n}\right)^{3}}=1+3 \sum_{\substack{n=1 \\ 9 \gamma_{n}}}^{\infty} \frac{n q^{n}}{1-q^{n}} .
$$

If we multiply by $\prod_{n=1}^{\infty} \frac{\left(1-q^{9 n}\right)^{3}}{\left(1-q^{n}\right)^{3}}$, we get

$$
\begin{aligned}
\prod_{n=1}^{\infty} \frac{\left(1-q^{3 n}\right)^{10}}{\left(1-q^{n}\right)^{6}} & =\prod_{n=1}^{\infty} \frac{\left(1-q^{9 n}\right)^{3}}{\left(1-q^{n}\right)^{3}}\left\{1+3 \sum_{\substack{n=1 \\
g \gamma_{n}}}^{\infty} \frac{n q^{n}}{1-q^{n}}\right\} \\
& =\frac{d}{d q}\left\{q \prod_{n=1}^{\infty} \frac{\left(1-q^{9 n}\right)^{3}}{\left(1-q^{n}\right)^{3}}\right\},
\end{aligned}
$$


or equivalently

$$
\int_{0}^{q} \prod_{n=1}^{\infty} \frac{\left(1-s^{3 n}\right)^{10}}{\left(1-s^{n}\right)^{6}} d s=q \prod_{n=1}^{\infty} \frac{\left(1-q^{9 n}\right)^{3}}{\left(1-q^{n}\right)^{3}}
$$

Recall the modular transformation for the Dedekind eta function, for example, see $[8$, Theorem 4.11], which may be written in the form

$$
q^{1 / 24} \prod_{n=1}^{\infty}\left(1-q^{n}\right)=\frac{1}{\sqrt{t}} p^{1 / 24} \prod_{n=1}^{\infty}\left(1-p^{n}\right)
$$

where $q=e^{-2 \pi t}, p=e^{-2 \pi / t}, \operatorname{Re}(t)>0$. If we take $t=1 / 3$, then $p=q^{9}$, and so in this case the modular transformation implies

$$
\begin{aligned}
q \prod_{n=1}^{\infty} \frac{\left(1-q^{9 n}\right)^{3}}{\left(1-q^{n}\right)^{3}} & =q \prod_{n=1}^{\infty} \frac{\left(1-p^{n}\right)^{3}}{\left(1-q^{n}\right)^{3}} \\
& =q\left(\frac{q}{p}\right)^{1 / 8}(\sqrt{t})^{3} \\
& =t^{3 / 2} \\
& =\frac{1}{3 \sqrt{3}} .
\end{aligned}
$$

Using this in (3.2) we complete the proof.

\section{REFERENCES}

[1] B.C. Berndt, Ramanujan's Notebooks, Part III (Springer-Verlag, New York, 1991).

[2] B.C. Berndt, Ramanujan's Notebooks, Part V (Springer-Verlag, New York, 1998).

[3] B.C. Berndt, S.H. Chan, Z.-G. Liu and H. Yesilyurt, 'A new identity for $(q ; q)_{\infty}^{10}$ with an application to Ramanujan's partition congruence modulo 11', Q.J. Math. 55 (2004), $13-30$.

[4] J.M. Borwein and F.G. Garvan, 'Approximations to $\pi$ via the Dedekind eta function', CMS Conf. Proc. 20 (1997), 89-115.

[5] J.M. Borwein and P.B. Borwein, 'A cubic counterpart of Jacobi's identity and the AGM', Trans. Amer. Math. Soc. 323 (1991), 691-701.

[6] J.M. Borwein, P.B. Borwein and F.G. Garvan, 'Some cubic modular identities of Ramanujan', Trans. Amer. Math. Soc. 343 (1994), 35-47.

[7] R. Chapman, 'Cubic identities for theta series in three variables', Ramanujan J. 8 (2004), 459-465.

[8] S. Cooper, 'Cubic theta functions', J. Comput. Appl. Math. 160 (2003), 77-94.

[9] H.M. Farkas and I. Kra, Theta constants, Riemann surfaces and the modular group, Graduate Studies in Mathematics 37 (Amer. Math. Soc., Providence, RI, 2001).

[10] N.J. Fine, Basic hypergeometric series and applications, Mathematical Surveys and Monographs 27 (American Mathematical Society, Providence, RI, 1988). 
[11] F. Garvan, 'Cubic modular identities of Ramanujan, hypergeometric functions and analogues of the arithmetic-geometric mean iteration', Contemp. Math. 166 (1994), 245-264.

[12] M. Hirschhorn, F. Garvan and J. Borwein, 'Cubic analogues of the Jacobian theta functions $\theta(z, q)$ ', Canad. J. Math. 45 (1993), 673-694.

[13] M. Hirschhorn, 'Three classical results on representations of a number', Sém. Lothar. Combin. 42 (1999). Art. B42f, 8 pp. (electronic).

[14] Z-G. Liu, 'Some Eisenstein series identities associated with the Borwein functions', Dev. Math. 4 (2001), 147-169.

[15] S. Ramanujan, 'On certain arithmetical functions', Trans. Camb. Phil. Soc. 22 (1916), 159-184. Reprinted in [17, 136-162].

[16] S. Ramanujan, Notebooks, (2 volumes) (Tata Institute of Fundamental Research, Bombay, 1957).

[17] S. Ramanujan, Collected papers (AMS Chelsea Publishing, Providence, RI, 2000).

[18] P. Solé, ' $D_{4}, E_{6}, E_{8}$ and the $\mathrm{AGM}$ ', in Applied algebra, algebraic algorithms and error-correcting codes (Paris, 1995), Lecture Notes in Computer Science 948 (Springer, Berlin, 1995), pp. 448-455.

Institute of Information and Mathematical Sciences

Massey University - Albany

Private Bag 102904

North Shore Mail Centre

Auckland

New Zealand

e-mail: s.cooper@massey.ac.nz 